Published in "International Journal of Modern Physics B 21(23-24):

4071-4075, 2007" which should be cited to refer to this work.

\title{
EFFICIENT ROUTING ON SCALE-FREE NETWORKS
}

\author{
TAO ZHOU \\ Department of Modern Physics, University of Science and Technology of China, \\ Hefei Anhui 230026, P. R. China \\ Department of Physics, University of Fribourg, Chemin de Muse 3, \\ CH-1700, Fribourg, Switzerland \\ zhutou@ustc.edu
}

\begin{abstract}
The nodes with the largest degree are very susceptible to traffic congestion, thus an effective way to improve traffic and control congestion can be redistributing traffic load in hub nodes to others. We proposed an efficient routing strategy, which can remarkably enhance the network throughput. In addition, by using detrended fluctuation analysis, we found that the traffic rate fluctuation near the critical point exhibits the $1 / f$ scaling in the power spectrum, which is in accordance with the empirical data.
\end{abstract}

Keywords: Traffic dynamics; scale-free networks; efficient routing; $1 / f$ noise.

Since the seminal works on the small-world phenomenon ${ }^{1}$ and the scale-free property ${ }^{2}$, the studies of complex networks have attracted a lot of interests within the physics community ${ }^{3}$. One of the ultimate goals of the current studies on complex networks is to understand and explain the workings of the systems built upon them ${ }^{4,5}$. The increasing importance of large communication networks, upon which our society survives, calls for the need for high efficiency in handling and delivering information. There have been many previous studies to understand and control traffic congestion on networks, with a basic assumption that the network has a homogeneous structure, however, many real-life communication networks, such as the Internet $^{6}$ and the World-Wide-Web ${ }^{7}$, have highly heterogeneous structure, thus it is of great interest to study the traffic flow on scale-free networks.

In this paper, we treat all the nodes as both hosts and routers ${ }^{8}$. The model is described as follows: at each time step, there are $\mathrm{R}$ packets generated in the system, with randomly chosen sources and destinations. It is assumed that all the routers have the same capabilities in delivering and handling information packets, that is, at each time step all the nodes can deliver at most $\mathrm{C}$ packets one step toward their destinations according to the routing strategy. We set $\mathrm{C}=1$ for simplicity. A packet, once reaching its destination, is removed from the system. We are most interested in the critical value $R_{c}$ where a phase transition takes place from free flow 

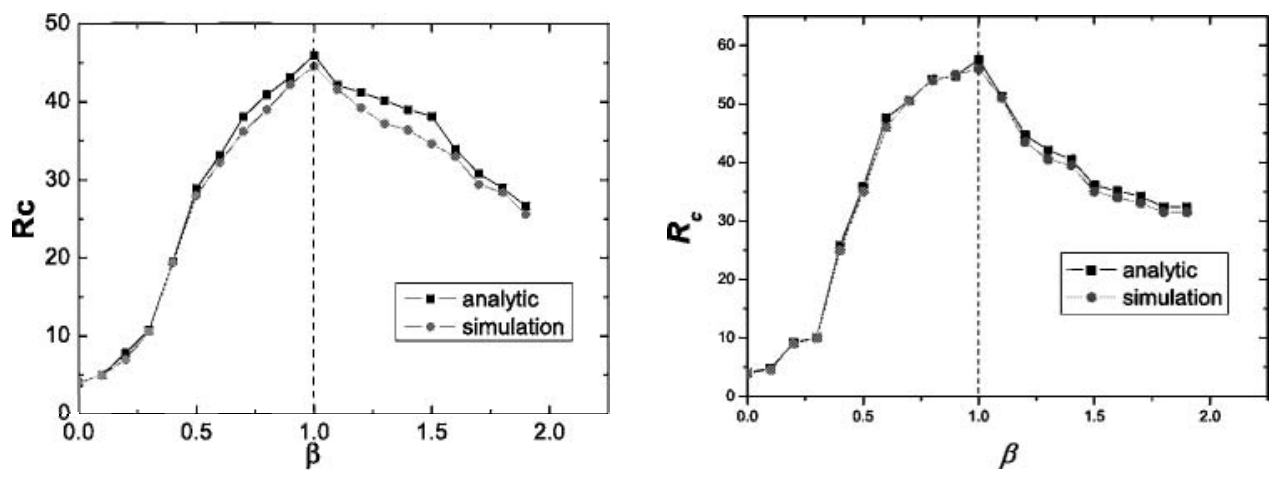

Fig. 1. (Color online) The critical $R_{c}$ vs $\beta$ for BA network with average degree 4 and of size $\mathrm{N}=1225$ (left panel) and $N=1500$ (right panel). Both simulation and analysis demonstrate that the maximal $R_{c}$ corresponds to $\beta \approx 1$. The results are the average over 10 independent runs.

to congested traffic. This critical value can best reflect the maximum capability of a system handling its traffic. In particular, for $\mathrm{R}<R_{c}$, the numbers of created and delivered packets are balanced, leading to a steady free traffic flow. For $\mathrm{R}>R_{c}$, traffic congestion occurs as the number of accumulated packets increases with time, simply for that the capacities of nodes for delivering packets are limited.

Consider the routing under global protocol ${ }^{9}$, where the whole structural information is available, and the fixed routing algorithm is the most widely used one for its obvious advantages in economical and technical costs ${ }^{10}$. Actually, the path with shortest length is not necessarily the quickest way, considering the presence of possible traffic congestion and waiting time along the shortest path. Obviously, nodes with larger degree are more likely to bear traffic congestion, thus a packet will by average spends more waiting time to pass through a high-degree node. All too often, bypassing those high-degree nodes, a packet may reach its destination quicker than taking the shortest path. In order to find the optimal routing strategy, we define the "efficient path". For any path between nodes $i$ and $j$ as $P(i \rightarrow j):=\mathbf{i} \equiv x_{0}, x_{1}, \cdots x_{n-1}, x_{n} \equiv j$, denote

$$
L(P(i \rightarrow j): \beta)=\sum_{i=0}^{n-1} k\left(x_{i}\right)^{\beta},
$$

where $k\left(x_{i}\right)$ denotes the degree of the node $x_{i}$, and $\beta$ is a tunable parameter. The efficient path between $\mathbf{i}$ and $j$ is corresponding to the route that makes the sum $L(P(i \rightarrow j): \beta)$ minimum. Obviously, $L_{\min }(\beta=0)$ recovers the traditionally shortest path length. As for any pair of source and destination, there may be several efficient paths between them. We randomly choose one of them and put it into the fixed routing table which is followed by all the information packets.

In Fig. 1, we report the simulation results for the critical value $R_{c}$ as a function of $\beta$ on Barabási-Albert (BA) network ${ }^{2}$ with the size $N=1225$ and $\mathrm{N}=1500$, 

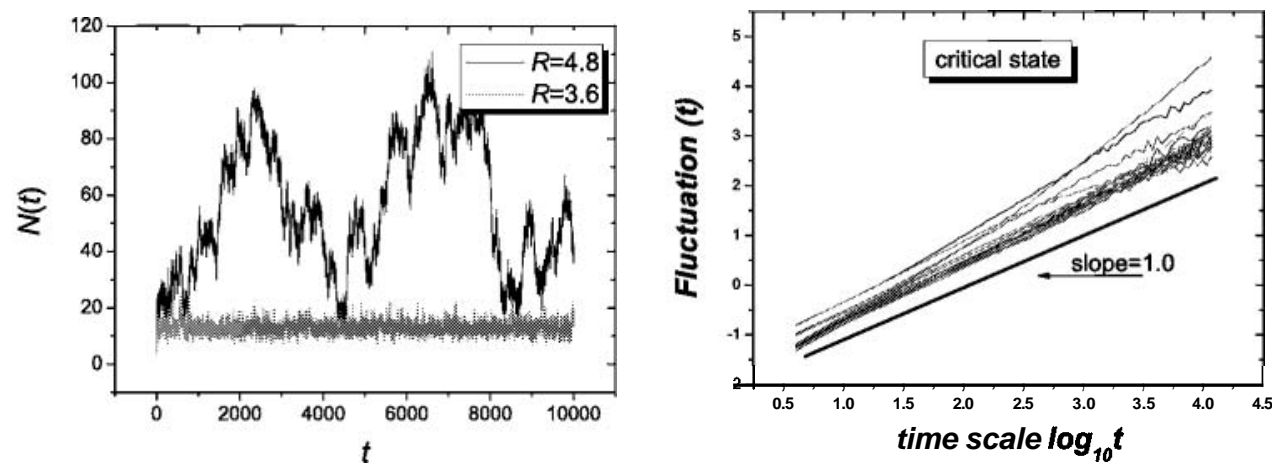

Fig. 2. (Color online) The time series of traffic load in critical and free state on a BA network with average degree 4 and of size $\mathrm{N}=1225$, where $N(t)$ denotes the number of packets at time $t$ (left panel). In this case, the critical value is $R_{c}=4.9$. The detrended fluctuation analysis of traffic rate process in critical states for different $\beta$ from 0 to 1.9 in step 0.1 (right panel).

which demonstrate that the optimal router strategy corresponding to $\beta=1$ and the size of BA network doesn't affect the value of optimal $\beta$. In comparison with the traditional routing strategy (i.e. $\beta=0$ ), the throughput $R_{c}$ of the whole network is greatly improved more then 10 times without any increase in algorithmic complexity. By extending the concept of betweenness centrality ${ }^{11,12}$ to efficient betweenness centrality $^{\mathrm{a}}$, the analytic results can be obtained according to the Little's law ${ }^{13,14}$. The analytical results are also shown in Fig. 1, which agree very well with the simulations.

Recently, the development of complexity science present a new point of view that is propitious for us to investigate the scaling behavior of network traffic. In particular, the empirical data of real traffic exhibit a $1 / \mathrm{f}$-type scaling in the power spectrum $^{15}$, which has also been reproduced by an ideal traffic model ${ }^{16}$. A typical simulation about the time series of the traffic load is shown in the left plot of Fig. 2, where the critical giant fluctuation can be seen when $\mathrm{R}$ is near $\mathrm{R}$,. To give a more accurate description of this statistical property, here we apply the detrended fluctuation analysis ${ }^{17}$ (DFA) on the traffic time series. It is seen from the right plot of Fig. 2, the scaling exponents, obtained by DFA for all $\beta$ are very close to 1.0, which strongly support the existence of self-similarity and long-range correlation in the traffic time series. This result accurately agrees with the empirical analysis on the real Ethernet traffic in which the corresponding exponent is $0.98^{18}$.

This studies may be useful for designing communication protocols for complex networks, considering there appears no increase in its algorithmic complexity. In addition, although the model presented here is very simple, it may catch hold of some essential features of real traffic as it can reproduce the $1 / \mathrm{f}$-type scaling behavior. It 
is worthwhile to emphasize that an analogical routing strategy based on local protocol, where only the local information is available for each router, can also greatly enhance the throughput of scale-free networks ${ }^{19,20}$. Especially, the correlation between a node's betweenness and its traffic load in the optimal case (i.e. $\beta=1$ ) of the current model is of the similar form to the relation between a node's degree and its traffic load in the case $\alpha=-1$ of that local traffic model ${ }^{19,20}$, thus we wonder if the $1 / \mathrm{f}$ scaling can also be detected in the local-routing system. More interesting, researchers have recently found some evidences indicating there may exist some common features among traffic dynamic, epidemic spreading and synchronizing process on scale-free networks. In susceptible-infected (SI) epidemics ${ }^{21,22,23}$, if the infectivity of each infected individual is limited, it is found that the contacting strategy preferring small-degree nodes predicts faster spreading ${ }^{24}$. And almost all the strategies used to improve network throughput and control congestion (e.g. to redistribute traffic load, to increase the capacities of hub nodes, to reduce the average distance, etc.), can also sharply enhance the network synchronizability ${ }^{25,26,27,28,29}$.

\section{Acknowledgments}

This work was partially supported by the National Natural Science Foundation of China under Grant Nos. 10635040, 70471033 and 10472116.

\section{References}

1. D. J. Watts, and S. H. Strogatz, Nature 393, 440 (1998).

2. A. -L. Barabási, and R. Albert, Science 286, 509 (1999).

3. R. Albert, and A. -L. Barabási, Rev. Mod. Phys. 74, 47 (2002).

4. M. E. J. Newman, SIAM Review 45, 167 (2003).

5. S. Boccaletti et al., Phys. Rep. 424, 175 (2006).

6. R. Pastor-Satooras, A. Vázquez, and A. Vespignani, Phys. Rev. Lett. 87, 258701 (2001).

7. R. Albert, H. Jeong, and A. -L. Barabási, Nature 401, 130 (1999).

8. T. Zhou, Dyn. Contin. Discret. Impuls. Syst. Ser. B-Appl. Algorithms 13, 463 (2006).

9. G. Yan, T. Zhou, B. Hu, Z. -Q. Fu, and B. -H. Wang, Phys. Rev. E 73, 046108 (2006).

10. C. Huitema, Routing in the Internet (Prentice Hall, NJ, 2000).

11. M. E. J. Newman, Phys. Rev. E 64, 016132 (2001).

12. T. Zhou, J. -G. Liu, and B. -H. Wang, Chin. Phys. Lett. 23, 2327 (2006).

13. R. Guimerá et al., Phys. Rev. Lett. 89, 248701 (2002).

14. L. Zhao, Y.-C. Lai, K. Park, and N. Ye, Phys. Rev. E 71, 026125 (2005).

15. K. Fukuda, H. Takayasu, and M. Takayasu, Physica A 287, 289 (2000).

16. B. Tadić, S. Thurner, and G. J. Rodgers, Phys. Rev. E 69, 036102 (2004).

17. C. K. Peng et al., Phys. Rev. E 49, 1685 (1994).

18. P. -L. Zhou et al., Proc. ICCCAS'06, pp. 2687-2691 (IEEE Press, 2006).

19. C. -Y. Yin et al., Phys. Lett. A 351, 220 (2006).

20. W. -X. Wang et al., Phys. Rev. E 73, 026111 (2006).

21. M. Barthélemy et al., Phys. Rev. Lett. 92, 178701 (2004).

22. T. Zhou, G. Yan, and B. -H. Wang, Phys. Rev. E 71, 046141 (2005).

23. T. Zhou, Z. -Q. Fu, and B. -H. Wang, Prog. Nat. Sci. 16, 452 (2006). 
24. T. Zhou et al., Phys. Rev. E 74, 056109 (2006).

25. A. E. Motter, C. Zhou, and J. Kurths, Phys. Rev, E 71, 016116 (2005).

26. M. Chavez et al., Phys. Rev. Lett. 94, 218701(2005).

27. M. Zhao, T. Zhou, B. -H. Wang, and W. -X. Wang, Phys. Rev. E 72, 057102 (2005).

28. T. Zhou, M. Zhao, and B. -H. Wang, Phys. Rev. E 73, 037102 (2006).

29. M. Zhao, T. Zhou, B. -H. Wang, Q. Ou, and J. Ren, Eur. Phys. J. B 53, 375 (2006). 